\title{
L'effet de la méthodologie sur la motivation des apprenants d'anglais de spécialité scientifique et technologique
}

Philip Hindley

\section{OpenEdition}

\section{Journals}

Édition électronique

URL : http://journals.openedition.org/asp/4400

DOI : $10.4000 /$ asp. 4400

ISSN : 2108-6354

\section{Éditeur}

Groupe d'étude et de recherche en anglais de spécialité

Édition imprimée

Date de publication : 1 mars 1993

Pagination : 509-518

ISSN : 1246-8185

\section{Référence électronique}

Philip Hindley, «L'effet de la méthodologie sur la motivation des apprenants d'anglais de spécialité scientifique et technologique », ASp [En ligne], 1 | 1993, mis en ligne le 04 juin 2014, consulté le 03 mai 2019. URL : http://journals.openedition.org/asp/4400 ; DOI : 10.4000/asp.4400

Ce document a été généré automatiquement le 3 mai 2019.

Tous droits réservés 


\title{
L'effet de la méthodologie sur la motivation des apprenants d'anglais de spécialité scientifique et technologique
}

\author{
Philip Hindley
}

\section{Introduction}

1 Comme beaucoup de professeurs d'anglais spécialisé, je me trouve souvent devant des étudiants qui sont obligés d'apprendre l'anglais parce qu'ils suivent un stage qui nécessite une connaissance de l'anglais. Mais il arrive souvent qu'ils ne soient pas motivés. Donc, ils n'apprennent pas, ou l'efficacité de l'apprentissage est diminuée à cause de ce manque d'enthousiasme chez l'apprenant.

2 La première question que je me pose est la suivante : «Que puis-je faire pour transformer mes étudiants démotivés en apprenants d'anglais très motivés?"; d'après moi, la solution à ce problème est dans le choix de la méthodologie. Il me semble que l'enseignant est beaucoup plus libre de choisir sa propre méthodologie dans sa salle de classe que de choisir, par exemple, un contenu qui est souvent déterminé par un programme fixe.

3 Voilà donc les raisons qui m'ont poussé à commencer cette recherche. La recherche ellemême comporte deux étapes : premièrement, une évaluation des manuels scientifiques et technologiques du point de vue des méthodologies sous-jacentes aux exercices et deuxièmement, des expériences auprès des étudiants pour trouver des exercices qui soient plus motivants que d'autres. 


\section{Évaluation des manuels}

4 Pour l'évaluation, j'ai commencé par examiner plusieurs manuels que j'ai trouvés dans divers instituts d'enseignement, bibliothèques et librairies en France et en Angleterre. Avec ces manuels, j'ai essayé de faire une taxonomie d'exercices selon les méthodologies sous-jacentes. Cette taxonomie est présentée en annexe 1.

5 En effet, dans ces manuels je n'ai trouvé que trois méthodologies principales: les méthodologies traditionnelle, audio-linguale et communicative. Par la méthodologie traditionnelle, j'entends tous les exercices basés sur la grammaire, les questions de compréhension, les traductions et les résumés. La méthodologie audio-linguale est beaucoup plus précisément définie et inclut des exercices structuraux. Enfin, la méthodologie communicative inclut tous les exercices de transfert d'information, les taches à résoudre et les exercices socio-linguistiques, y compris les dialogues et les simulations.

6 Je voudrais évoquer ici quatre points qui me semblent importants pour ma recherche et qui se sont présentés pendant l'évaluation des manuels. Tout d'abord, dans 22 manuels sur 27 on trouve des exercices de transfert d'information, y compris souvent un transfert entre des graphiques ou des schémas, à l'écrit ou à l'oral. Il me semble qu'une telle activité est très utile pour un scientifique qui, par exemple, est assez souvent obligé d'interpréter un graphique ou un schéma.

7 Deuxièmement, j'ai remarqué que les exercices sociolinguistiques étaient très peu nombreux. Pourtant, ces exercices ne sont pas sans importance dans le domaine de l'anglais spécialisé scientifique et technologique.

8 Troisièmement, j'ai trouvé beaucoup d'exercices convenant à la phase de la mise en pratique ou du réemploi et de la production semi-guidée, mais très peu d'exercices convenant à la phase de la production libre. Cependant, il est très important qu'un apprenant passe par toutes les phases, de la présentation jusqu'à la production libre. La dernière phase met la langue apprise en contexte, et elle est donc la phase la plus difficile à réaliser par l'apprenant et la seule indication qu'il/elle a vraiment acquis les éléments enseignés.

9 En dernier lieu, j'ai eu le sentiment que les exercices communicatifs n'allaient pas assez loin; qu'ils avaient besoin d'être développés. En effet, un exercice communicatif ne consiste pas seulement à mettre la langue en contexte en faisant, par exemple, des exercices de transfert d'information, mais un exercice communicatif doit aussi donner une raison pour apprendre. C'est à dire qu'il fixe un but que l'apprenant doit atteindre et qui n'est pas forcément linguistique, les objectifs linguistiques étant atteints pendant la réalisation de ce but.

10 Mais tous les points que je viens d'évoquer ne sont que des généralisations, l'évaluation m'a fourni une grande gamme d'exercices et d'idées pour créer des exercices pour la phase expérimentale.

\section{Phase expérimentale}

11 Au cours de la phase expérimentale, je travaille sur la motivation de l'apprenant en fonction de la méthodologie employée par l'enseignant. J'enseigne l'anglais à deux types 
d'apprenants. Les premiers sont des élèves d'un collège bilingue qui suivent des cours d'anglais spécialisé pour comprendre des cours de physique en anglais, et les seconds sont des étudiants préparant un D.U.T. (diplôme universitaire de technologie) d'informatique et de gestion.

Pendant les expériences, je distribue aux apprenants divers exercices et, après chaque activité, je note leur avis sur le plaisir qu'ils ont pris à cet apprentissage. J'utilise l'échelle Likert selon laquelle ils indiquent leur avis en mettant un chiffre compris entre 0 et 10 (voir figure 1).

Figure 1. Questionnaire distribué aux apprenants.

Answer the following question by placing a number from 0 to 10 next to it.

0 indicates not at all and 10 a lot

how much did you enjoy this activity?

13 J'additionne les totaux pour chaque classe et je calcule « un indice de motivation » pour chaque exercice en convertissant les totaux en pourcentage. En étudiant ces indices, il est possible de voir quels exercices sont les plus motivants. Ensuite, je fais les moyennes de tous les indices pour trouver des catégories d'exercices plus motivants.

14 Pour l'instant, je suis dans la phase expérimentale mais l'analyse déjà faite suggère que les exercices les plus motivants sont ceux qui ont une nature communicative. L'indice de motivation moyenne est de 68 pour les exercices communicatifs et il n'est que de 45 pour les autres exercices non-communicatifs (voir tableau 1).

Tableau 1. Indices de motivation des divers exercices

\begin{tabular}{|l|l|}
\hline \multicolumn{1}{|c|}{ TYPE D'EXERCICE } & INDICE DE MOTIVATION \\
\hline TRADITIONNELS & \\
\hline (a) basés sur la grammaire & 47 \\
\hline (b) exercices de conversion & 51 \\
\hline (c) exercices à trous & 39 \\
\hline $\begin{array}{l}\text { (d) construction de phrases avec un } \\
\text { lexique donné }\end{array}$ & 40 \\
\hline (e) questions de compréhension & 42 \\
\hline (f) résumés & 37 \\
\hline (g) traductions & 47 \\
\hline AUDIO-LINGUAUX & \\
\hline exercices structurauX & 55 \\
\hline COMMUNICATIFS & \\
\hline (a) transfert d'information & 74 \\
\hline (b) résoudre des tǻches & 62 \\
\hline (c) socio-linguistlques & 68 \\
\hline EXERCICES NON-COMMUNICATIFS & 45 \\
\hline EXERCICES COMMUNICATIFS & 68 \\
\hline
\end{tabular}




\section{Observations}

15 Je reviens sur les observations qui me semblent les plus importantes dans l'évaluation des manuels. Il existe trois aspects dans un exercice : le contenu (grammatical, lexical, etc.), le type d'exercice (exercice à trous, exercice de conversion, etc.) et l'approche que l'enseignant choisit pour faire passer l'exercice. Plutôt qu'une méthodologie sous-jacente aux exercices, il est plus exact de dire, qu'il existe des types d'exercices qui conviennent à une méthodologie mieux qu'à une autre, mais une méthodologie apparaît vraiment dans l'approche choisie par l'enseignant. Cette approche peut être qualifiée de microméthodologie.

16 Quand on parle de micro-méthodologie communicative, il est bon de distinguer deux éléments dans cette approche ; d'une part le but fixé par l'enseignant et, d'autre part, les méthodes que l'enseignant met à la disposition de l'apprenant pour que celui-ci puisse atteindre ce but.

17 Par exemple, dans une simulation que j'ai adaptée du manuel GOTO: anglais informatique pour communiquer de Déchet (1988) l'enseignant divise la classe en groupes de deux personnes, la moitié des groupes étant des vendeurs d'ordinateurs et l'autre moitié des acheteurs pour des entreprises. Les acheteurs rendent visite à tous les vendeurs afin de choisir un ordinateur pour leur entreprise.

18 À la fin de cette simulation, on trouve les vendeurs qui ont vendu le plus grand nombre d'ordinateurs. Une analyse de ces exercices pour les vendeurs et les acheteurs est donnée dans la figure 2.

Figure 2. Analyse de l'exercice vendeurs/acheteurs

(a) VENDEURS

\begin{tabular}{|c|c|}
\hline $\begin{array}{l}\text { TRANSFERT D'INFORMATION } \\
\text { (parler aux acheteurs) }\end{array}$ & $\begin{array}{l}\text { MÉTHODES EMPLOYÉES } \\
\text { par l'apprenant }\end{array}$ \\
\hline $\begin{array}{l}\text { GAGNER } \\
\text { (vendre le plus grand } \\
\text { nombre d'ordinateurs } \\
\text { possible) }\end{array}$ & BUT \\
\hline
\end{tabular}

(b) ACHETEURS

\begin{tabular}{|c|c|}
\hline \multicolumn{2}{|l|}{$\begin{array}{l}\text { RECUEILLIR UNE } \\
\text { INFORMATION } \\
\text { (sur les ordinateurs en option) }\end{array}$} \\
\hline & $\begin{array}{l}\text { MÉTHODES EMPLOYÉES } \\
\text { PAR L'APPRENANT }\end{array}$ \\
\hline \multicolumn{2}{|l|}{$\begin{array}{l}\text { DISCUTER } \\
\text { (pour choisir un ordinateur) }\end{array}$} \\
\hline $\begin{array}{l}\text { OBTENIR UN CONSENSUS } \\
\text { (choisir un ordinateur) }\end{array}$ & BUT \\
\hline
\end{tabular}


19 Nous donnons souvent aux apprenants un exercice « communicatif » auquel il manque un but bien défini. Je ne considère pas, par exemple, qu'un exercice de transfert d'information ou un dialogue peut être qualifié d'exercice communicatif s'il lui manque une vraie raison pour être fait. Dans l'exemple d'un exercice de Donovan (1978), il existe la possibilité de créer un exercice communicatif en ajoutant un but (voir figure 3).

Figure 3. Analyse de l'exercice de Donovan (1978)

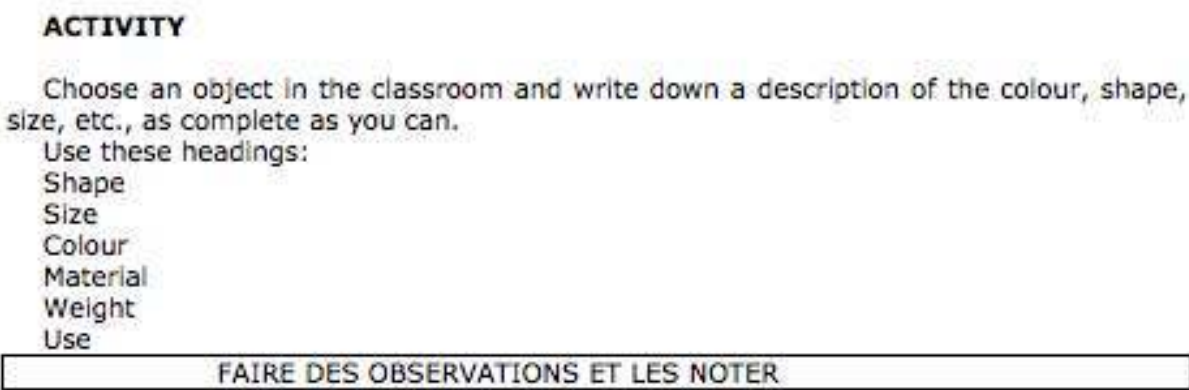

Describe your object to the class and see if they can find out what it is. TRANFERER UNE INFORMATION

En ajoutant un but, l'exercice a un sens, une raison pour être fait, et devient donc un vrai exercice communicatif.

\section{Conclusion}

21 Il m'est très difficile aujourd'hui de formuler une conclusion, mais je voudrais continuer mes expériences afin de prouver l'importance qu'il y a à donner un but aux exercices pour les rendre plus motivants pour l'apprenant. Pour l'instant, je suis en train d'établir une liste de méthodes, et une liste de buts qui peuvent être combinés dans une microméthodologie et servir d'éléments moteurs pour l'apprenant (voir tableau 2). 
Tableau 2. Méthodes et buts qui peuvent être combinés dans une micro-méthodologie

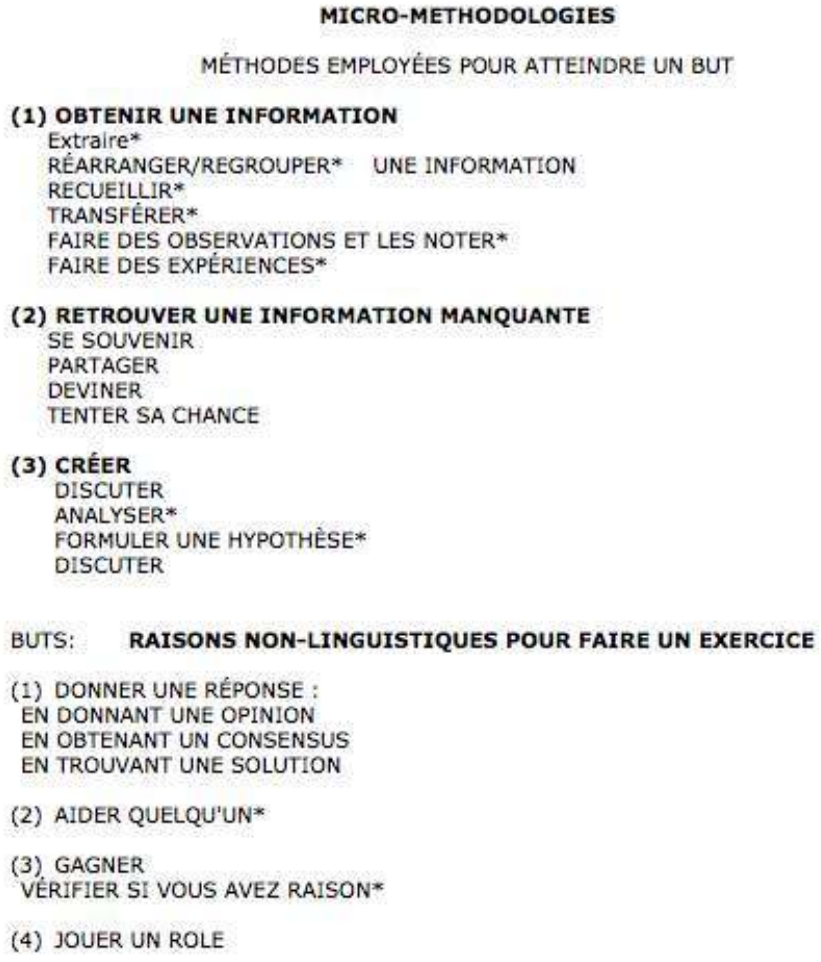

(4) JOUER UN ROLE

Les méthodes et les buts marqués d'une astérisque sont considérés comme plus adaptés aux scientifiques.

Les listes données sur ce tableau sont encore incomplètes et quelques idées s'imbriquent. Néanmoins, ces listes peuvent servir de point de départ à une réflexion ultérieure. Je peux déjà dire qu'il existe un potentiel de motivation à récupérer pour la langue de spécialité scientifique et technologique, en particulier si on utilise les méthodes et les buts adaptés aux façons de penser et de travailler des scientifiques.

\section{BIBLIOGRAPHIE}

\section{Liste des manuels évalués}

Adamson. D. et M. Bates. 1977. Nucleus English for Science and Technology. Biology. Londres : Longman.

Allen, J.P.B. et H.G. Widdowson. 1979. English in Physical Science. Oxford : Oxford University Press. A.S.T.E.L. 1971. Computer Men. Paris : Didier.

Bates, M. et T. Dudley-Evans. 1983. General Science. Londres : Longman.

Blackie, D. 1985. English for Basic Physics. Surrey : Thomas Nelson. 
Bolitho, A. R. 1983. Learn English for Science. Londres : Longman.

Brookes, B. C. (dir.). 1979. Scientifically Speaking. Londres : BBC.

Brookes, M. et F. Lagoutte. 1989. English for Information Technology. Paris : Belin.

Chaplen, F. 1988. A Course in Intermediate Scientific English. Londres : Unwin Hyman.

Dechet, A. 1988. GOTO L'Anglais informatique pour communiquer. Paris : Ellipses.

Donovan, P. 1978. Basic English for Science. Oxford : Oxford University Press.

Dudley-Evans, T. 1989. Writing Laboratory Reports. Surrey : Nelson.

Ewer, J. R. et G. Latorre. 1970. A Course in Basic Scientific English. Londres : Butler and Tanner.

Glendinning, E. H. et J. McEwan. 1989. English in Computing. Walton-On-Thames : Thomas Nelson.

Hic, S. 1991. English for Information Systems. Hertfordshire : Prentice Hall.

Kay, V. 1985. Biological Sciences: Developing Reading Skills in English. Oxford : Pergamon.

Keegel, J. C. 1976. The Language of Computer Programming in English. New York : Regents.

Morrow, K. 1980. Skills for Reading with Extracts from the New Scientist. Oxford : Oxford University Press.

Mullen, N. D. et P.C. Brown. 1983. English for Computer Science. Oxford : Oxford University Press. Nogas, G.D. et A.R. Bolitho. 1986. Start English for Science. Essex : Longman.

Robinson, P. 1972. Special English Computer Programming. Londres : Collier Macmillan.

Robinson, P. 1987. Computers Cope Better. Middlesex : Nelson.

Royds-Irmak, D. E. 1975. Beginning Scientific English: Book 1 and 2. Middlesex : Nelson.

Swales, J. 1971. Writing Scientific English. Surrey : Nelson.

Templeton, H. 1983. English for Mechanical Science. Oxford : Oxford University Press.

Upjohn, J., S. Blattes et V. Jans. 1991. Minimum Competence in Scientific English. Grenoble : Presses Universitaires de Grenoble.

Walker, T. 1989. Computer Science. Londres : Cassell.

\section{AUTEUR}

\section{PHILIP HINDLEY}

IUT de Villetaneuse Paris 13. philip.Hindley@univ-paris1.fr 